\title{
Vierzigtägige Retention des Kopfes eines reifen Kindes sammt Placentarresten innerhalb der Ge- bärmutterhöhle ohne jedwede Reaction.
}

\author{
Ein Beitrag über die Toleranz der Gebärmutter gegen \\ traumatische und septische Einwirkungen. ${ }^{1}$ )
}

Von

Alois Valenta.

Am 13. Februar $1882^{1}$ ) ward lant Prot.-Nr. 31 die Eisenbahnarbeitersfrau Marie S... in Snēberje, eine kleine halbe Stunde von Laibach wohnhaft, auf die Gebärklinik gebracht mit dem Bemerken, es sei noch seit dem 8. Januar der von einem Arzte abgeschnittene Kindskopf in ihrem Leibe, und da weder sie noch ihre Umgebung es länger ob des Gestankes, welchen der Ausfluss aus ihren Genitalien verursache, auszuhalten vermögen, so bitte sie, man wolle ihr denselben ehethunlichst unter jeder Bedingung entfernen.

Ich hatte zwar schon gerüchtweise von dem Unglüclisfalle gehört, konnte jedoch meinen Sinnen fast nicht tracen, dass diese Person noch leben sollte, ja dass es eben diese sich vorstellende Frau sei, welche einen Puls von 72 bis 78 Schlägen, eine normale Temperatur, eine reine Zunge, laut Aussage guten Appetit und gesunden Schlaf darbot, welche thatsächlich noch den Kindskopf in sich stecken haben soll, wenn nicht der colossale, aashafte Geruch, den dieselbe verbreitete, schon im vorhinein die Wahrheit des Gesagten hand-, "will sagen nasengreiflich bestätigt hätte.

1) Vorgetragen im Vereine der Aerzte in Krain zu Laibach. 
432 Valenta, Vierzigtägige Retention des Kopfes eines reifen Kindes

Die alsbald vorgenommene Untersuchung liess einen über kindskopfgrossen, eigenthümlich knitternden, unbedeutend empfindlichen Tumor über der Schoossfuge erkennen, welcher sich bimanuell als der antevertirte Uterus manifestirte, dessen Vaginalportion vollkommen rückgebildet, derbhart erschien; das ectropiale Orificium externum liess den untersuchenden Finger auf circa $2 \mathrm{~cm}$ in den Halskanal vordringen und durch den für die Fingerspitze nicht durchgängigen inneren Muttermund war deutlich ein herabragender scharfkantiger Körper - eine Knochenspitze erkennbar, and wenn man die Gebärmutter nach abwärts schob, resp. deren Grund eindrückte, konnte man das oben erwähnte Knittern deutlich fühlen, ja hören, und es ergoss sich dann dabei gleichzeitig in reichlichem Maasse ein dunkelbraunes, pestilentialisch stinkendes Secret; - somit ein Befund, welcher weiter keinen Zweifel mehr über die Richtigkeit der Angabe zuliess.

Frau S..., eine 35jährige robuste, in jeder Richtung gut gebaute IVpara, war stets gesund gewesen bis vor acht Jahren, wo sie sich durch Verkühlung eine Schwerhörigkeit zuzog, welche dermalen bereits hart an Taubheit grenzt. Ihre drei ersten Schwangerschaften resp. Geburten überstand sie regelrecht, die letzte, i. e. vierte Schwangerschaft, hatte am 8. Januar ihr rechtmässiges Ende erreicht. Da sich bei der Geburt resp. nach dem Fruchtblasensprunge das Kind in einer Schulterlage präsentirte, wurde ein Geburtsarzt beigezogen, welchem endich nach langer und schwieriger Arbeit, nachdem or vorher den vorliegenden Arm enueleirt habe, die Wendung auf den Fuss - oder beide Füsse? - und die Extraction des Rumpfes bis zum Kopfe gelungen sei. Trotz aller Versuche ${ }^{1}$ ) ging die Entwickelung des letzteren nicht vor sich, weshalb er denselben einfach - warum? - vom Rumpfe abgeschnitten "und alsdann die Gobärende verlassen habe, ohne sich dann weiter mehr um selbe zu kümmern!

Zwei anderen herbeigerufenen Aerzten gelang die Entwickelung des abgeschnittenen Kopfes auch nicht; wie ich nachträglich in Erfahrung gebracht, haben dieselben nur Ergotin (sic!) verschrieben. Da der Ehemann von einer Uebertragung der Kreissenden ins Laibacher Gebärhaus nichts hören wollte, wurde die Frau nun einfach sich selbst ïberlassen, und es sei gar nichts anderes

1) Wie ich erfahren habe, soll die Perforation und Extraction mittels des Kranioklaster nicht versucht worden sein. 
vorgenommen worden, als eine Uebertragung derselben von einem Bette ins andere, um die von der massenhaft abfliessenden Jauche durchtränkten Unterlagen entfernen zu können. - Bis zum achten Tage nach der Operation ward die Gebärende gar nicht ausgespritzt; von diesem Tage an soll eine zweite beigezogene Hebamme so jeden zweiten bis dritten Tag mit einfachem lauen Wasser sie ausgespritzt haben; - dessenungeachtet habe sie nie einen Schüttelfrost gehabt, auch sonst keine besonderen Bauchschmerzen verspürt, es seien der Appetit, die Darm- und Harnentleerungen und der Schlaf ganz regelrecht geworden, nur schwach fühlte sie sich und der Wochenfluss war unausstehlich ob seines Gestankes. Die Frau wollte sich schon früher ins Gebärhaus ïbertragen lassen, jedoch ihr Mann hatte es nie zugegeben, bis der k. k. Bezirksarzt Dr. Kapler, intervenirend, deren Transferirung dorthin ex officio verfügte; so lautete die äusserst interessante Anamnese.

Da nach dem oben Erwähnten - Puls 72, Temperatur 37,5 C. - keine Gefahr im Verzuge war, vertröstete ich die Aermste, um einerseits Zeit zur Beobachtung zu gewinnen und andererseits über das weiter Vorzunehmende klar zu werden, mit der Möglichkeit, dass der Kopf ohne Operation abgehen werde und wir diesbezügliches $\mathrm{zu}$ versuchen verpflichtet seien. - Sie wurde demgemäss dreistündlich mit einer 3proc. Carbollösung ausgespritzt, bekam Sitzbäder und Tag und Nacht blieb der Leiter'sche Wärmeregulator auf den Unterleib aufliegend.

Alles vergeblich - es blieb Alles, allgemein und örtlich, in statu quo, es trat weder eine allgemeine noch eine örtliche Reaction ein, die Frau erfreute sich - sit venia verbo - eines zufriedenstellenden allgemeinen Wohlbefindens, und örtlich i. e. an der Gebärmutter gab sich nicht die geringste Spur einer erwachenden Contractilität - Wehenthätigkeit - kund, deren auffällig dünne Wandungen waren wie ein Zeltdach über den darin steckenden Schädel gespannt. - Dieser anatomische als physiologische Befund stellte somit die Thatsache fest, dass wir es hier mit einer vollkommen puerperal involvirten Gebärmutter zu thun hatten, deren Grund und Körper durch den fremden Inhalt passiv ausgedehnt erhalten blieb, während sich deren Hals, baar des Hindernisses, anstandslos activ rückbilden konnte; ein Befund, welcher mich, offen gesagt, nicht das Beste bezüglich des glatten Gelingens der Extraction des Kopfes hoffen liess, um 
so mehr, als auch nach diesen Erfahrungen das Wie? der Operation sehr fraglich erschien, indem unter so bewandten Umständen auf eine erfolgreiche Auregung resp. Wiedererwachen der physiologischen Thätigkeit der Gebärmutter, i. e. Hervorrufung von Wehen, nicht viel zu rechnen war. Der Versuch, letztere anzufachen, erschien dennoch als das Rationellste, um der Conditio sine qua non der möglichen Entwickelung des Kopfes, nämlich dem Offenmachen des Halskanales resp. des Muttermundes zu entsprechen; somit wurde beschlossen, da die Leidende uns fort und fort bestürmte, sie zu erlösen, durch gleichzeitige mechanische Dilatation und innerliches Verabreichen von Secale cornutum die Operation einzuleiten und dann durchzuführen.

Der Operationsverla uf war folgender: Am 15. Februar um 5\% Uhr Abends wurde nach vorheriger tüchtiger Irrigirung mit einer 3 proc. Carbolsäurelösung ein der Durchgängigkeit entsprechender Pressschwamm bis zum inneren Muttermund eingelegt und bis Abends 10\% Uhr liegen gelassen. Nach dessen Entfer nung wurde nun ein Tupelostift von $1 \mathrm{~cm}$ Dicke eingefïhrt und derselbe den 16. Februar, Morgens $6 \mathrm{Uhr}$, durch einen fast $2 \mathrm{~cm}$ dicken cylindrischen Pressschwamm vertauscht, welcher bis zum eigentlichen Operationsbeginne, i. e. bis $10 \%$ Uhr Vormittags liegen blieb.

Gleichzeitig wurden fünf Dosen von frisch pulverisirtem Secale cornutum à $1 \mathrm{~g}$ um 2,3, 4, 6 und 7 Uhr Morgens verabreicht. - Nach jedesmaligem Wechsel der Quellmeissel ergoss sich massenhafte Jauche und bei der gleich nachfolgenden resp. vorherigen Carbolsäureirrigation gingen stets kleine Knochenfragmente $a b$, jedoch gab während der ganzen Manipulation die Patientin weder subjectiv wehenartige Schmerzen an, noch wurde objectiv ein zeitweiliges Härterwerden der Gebärmutter wahrgenommen, dieselbe blieb stets elastisch weich. Nachdem die Chloroformnarkose perfect geworden war, wurde, wie gesagt, um $10^{\mathrm{J} / 2}$ Uhr der Pressschwamm entfernt, ausgespritzt und bei einer Weite des nunmehr kürzer gewordenen Cervicalkanales von circa $2,5 \mathrm{~cm}$ und des $1,5 \mathrm{~cm}$ messenden, straff ringförmigen inneren Muttermundes zur Extraction geschritten. Mittels einer langen gekreuzten Polypenzange mit festem Schlosse wurden nun nach und nach zuerst die Basalknochen, dann die Schläfebeỉne, das Stirnbein und zuletzt die beiden Seitenwandbeine mïhsam entfernt; selbe mussten stets zerbröckelt werden, um sie durchzubringen. Bo- 
sonders schwierig war die Entwickelung der Ossa parietalia, indem dieselben zeltförmig innig an den Gebärmuttergrund angelagert resp. angeschmiegt waren. - Man musste, den Gebärmuttergrund flaschenbodenartig eindrüickend, diese platten Knochen zuerst einknicken, dann erst konnte man mit dem Zeigefinger dieselben abheben und hebelartig einen Knochenrand gegen den Muttermund so herabbewegen, dass der Knochen von der Zange gefasst und auf selbe gleichsam aufgerollt und entfernt werden konnte, freilich erst dann, nachdem man nach rechts und links hinten den eine circa $4 \mathrm{~cm}$ breite Querspalte bildenden, äusserst straff sich spannenden äusseren Muttermund incidirte, um eine Zerreissung desselben durch die Knochenränder zu verhïten, in der richtigen Voraussetzung, es sei unter den obwaltenden Umständen bezüglich der Ansteckungsgefahr jedenfalls eine glatte Schnittwunde einer zerfetzten Risswunde vorzuziehen.

$\mathrm{Zu}$ bemerken ist, dass mit den Knochenstücken stets kleine, ziemlich frisch aussehende Placentarreste abgingen, und siehe da, nachdem endlich um 12 Uhr Mittags alle Knochenfragmente - über 40 an der Zahl - entfernt waren, fand man links unten noch einen kleinhandtellergrossen, innig anhaftenden Placentarrest vor, welcher mit dem Fingernagel und Schablöffel thunlichst abgekratzt resp. entfernt wurde, worauf sich jedoch leider ein ziemlicher Blutabgang einstellte.

Selbstverständlich wurde die Uterushöhle während der Operation wiederholt mit einer lauen 3 proc. Carbolsäurelösung ausgespritzt, und nach der Operation wurde ob der Blutung mit Erfolg eine ausgiebige intrauterine Heisswasserinjection (von $+50^{\circ} \mathrm{C}$. und 2 proc. Carbolsäure) vorgenommen, und schliesslich oben im Schoosse eine subcutane Ergotininjection gemacht.

Ueber den Verlauf des nunmehrigen - sit venia verbo - zweiten Wochenbettes ist eigentlich nichts oder auch sehr viel zu erwähnen, dasselbe verlief ohne jedwede Reaction. Die Wöchnerin bekam die ersten Tage erregende Umschläge, wurde täglich einige Male mit 2 proc. Carbolsäurelösung intrauterin ausgespritzt, und das Facit war resp. blieb, dass die Temperatur niemals über 37,5 stieg, der Puls stets zwischen 72 bis 78 Schläge zählte, dass sich nicht die geringste Empfindlichkeit der Gebärmutter kundgab, die Lochien alsbald normal wurden, Schlaf, Appetit und die sonstigen Verrichtungen sich normal verhielten, kurzum so, dass die Wöchnerin, welche übrigens schon 
am vierten Tage aufstehen wollte, am 8. März das erste Mal aufstand und am 12. März gesund entlassen werden konnte. Bei ihrer Entlassung war der Uterus um kaum $1 \mathrm{~cm}$ vergrössert, beweglich, normal antevertirt, die Vaginalportion vollkommen rückgebildet, das Orificium externum geschlossen; die Secretion geruchlos, äusserst gering, nirgends in der Umgebung irgend eine Spur von Empfindlichkeit wahrnehmbar.

Epikritische Bemerkungen. Unser Fall ist in doppelter Beziehung äusserst überraschend, 1) ob der Toleran $z$ der Gebärmutter, dass dieselbe so lange einen fremden, ausser jeder Verbindung mit ihr stehenden Körper in sich duldete, i. e. nicht die geringste Spur einer Thätigkeit zu dessen Ausstossung kundgab, and 2) dass unter solchen Verhältnissen keine Septicaemie auftrat.

Was die Toleranz der Gebärmutter anbelangt gegenüber einem solchen leblosen Inhalte, so ist diesbezüglich die mit Bienenfleiss zusammengestellte Arbeit von Carl Liebmann ${ }^{1}$ ) sehr lehrreich, nur handelt dieselbe von solchen Früchten resp. Eiern, welche während der Schwangerschaft, i. e. vor deren physiologischem Ende, abgestorben, alsdann noch durch längere Zeit im Uterus verblieben sind. Liebmann meint, die Retentionsursache in derlei Fällen sci in einer mangelhaften Erregung jener Theile des Centralnervensystemes, welche die Wehenthätigkeit regeln, zu suchen; diese mangelhafte Erregbarkeit kann nun eine Folge von Veränderungen des Centrums selbst sein, oder es können die leitenden Nervenbahnen durch Anomalie der Gesammternährung specielle Reize schwächer oder gar nicht zum Centralorgane leiten. Wird auf diese Weise der mumificirte Fötus, resp. das abgestorbene Ei im Uterus als fremder Körper, frei von jeder organischen Verbindung mit dem mütterlichen Organismus, dem Einflusse des Centralnervensystems entzogen, eine Zeit lang ertragen, giebt es gar keinen Grund anzunebmen, dass dieser Zustand nicht eine unbestimmt lange Zeit, ja immer fortdavern könne - intrauterine Lithopädiumbildung -, und demgemäss könnte man sogar noch weiter gehen und anneh-

1) Beitrag zur Frage von der Retention abgestorbener Früchte in der Gebärmnttex, veröffentlicht in den Beiträgen zur Geburtshülfe und Gynäkologie in Berlin 1874, III. Bd., S. 47. 
men, dass die Ausstossung einer Frucht, welche länger als neun Monate in der Gebärmutter beherbergt wurde, nur durch Hinzutreten äusserer Momente, welche dem Retentionsprocesse fremd sind - missed labour - erfolge, denn es ist gar nicht einzusehen, warum sich der Uterus, der seinen leblosen Inhalt so lange geduldig ertragen hat, plötzlich desselben zu entledigen suche.

Den besten thatsächlichen Beweis der Richtigkeit letzterer Praemissen giebt Freund's , Geschichte einer Frau, welche den vor zehn Jahren abgerissenen Kindskopf noch heute in einzelnen Resten im Uterusträgt" 1 ), und einen weiteren diesbezüglichen eclatanten Beitrag liefert eben unser Fall selbst, denn auch hier zeigte der Uterus nicht die geringste Neigung zur Ausstossung des fremden Inhaltes, ja selbst dann noch nicht, als man denselben zur Wehenthätigkeit künstlich anzuregen versuchte, und ich bin vollkommen überzeugt, dass die Person den Kopf noch Monate, ja möglicherweise auch noch Jahre weiter anstandslos getragen hätte, wenn nur eine systematische Reinigung durch Ausspritzungen und Bäder in Anwendung gekommen wäre.

Was die Hauptursache dieser anhaltenden Unthätigkeit der Gebärmutter in meinem Falle betrifft, bin ich jedoch dieser, allerdings auch nicht erwiesenen Ansicht, dass dieselbe, wie bereits früher erwähnt wurde, in der vollständig z u Stande gekommenen puerperalen Involution der Gebärmutter liegen dürfte. - Thatsächlich war der Gebärmutterhals vollkommen rückgebildet, nämlich bezüglich seiner Stärke, Consistenz und dergleichen. Körper und Grund waren jedoch durch den Kopf am physiologischen Rückbildungsprocesse behindert, sie involvirten sich auch centripetal, mussten aber passiv ausgedehnt bleiben, Beweis dessen ihre Dünnheit, und eben deshalb, nämlich wegen dieser vollendeten puerperalen Involution, gab die Gebärmutter auch sowohl in der Ruhe als in der künstlichen Anregung keine Geneigtheit zu Contractionen, resp. zur Expulsion ihres fremden Inhaltes, i. e. des Kopfes kund. Ich möchte diesen Fall theilweise unter die Kategorie der ", missed labour" benannten Fälle einreihen, allerdings wäre "artificial" beizusetzen.

1) Freund: Ueber Toleranz des weiblichen Genitalkauales gegen traumatische und septische Einwirkungen. Deutsche Kilinik 1869, Nr. 33. 
438 Valenta, Vierzigtägige Retention des Kopfes eines reifen Kindes

Das zweite Moment, dass sich nämlich nicht eine Spur voul Septicaemic kundgab, erachte ich jodoch für das noch interessantere, aber auch räthselhaftere. Wenn man bedenkt, dass oft ein schmutziger untersuchender Finger oder ein unreines Instrument genügt, um einer Gebärenden, resp. einer normal entbundenen Wöchnerin den Todeskeim einzuimpfen, um so mehr bleibt dies in unserem Falle, wo derartige Eingriffe, wie eine schwierige Wendung nach vollführter Armenucleation und darauffolgende, sicherlich nicht selur zarte Extractionsvel'suche gewiss nicht olne jedwede Verletzung, mindestens der Schleimhaut, abgelaufen sein dürften, unerklärlich, dass demnoch keine Blutvergiftung durch dieses aashafte, ja cadaveröse Secret stattgefunden habe _. - grau ist alle Theorie _. _ - kann man in unserem Falle mit vollstem Rechte sagen! Nicht minder merkwürdig bleibt auch trotz der intercurrirenden flissigen Carbolsäureirrigationen, dass die von mir bei der Extraction zweifelsohne von den Knochensplittern gesetzten vielfachen, wenn auch kleinen Schleimhautrisse, ja die aus obigen Gründen unausweichlich nothwendig gewordenen Schnittwunden nicht ebenso Pforten zur septischen Blutvergiftung abgegeben haben.

Eine unmaassgebliche Exklärung dieses Räthsels möchte ich in Folgendem zu finden glauben, nämlich in dem ursprünglichen Tetanus uteri und der darauf folgenden, stetig fortschreitenden puerperalen Involution desselben.

Die sowohl traumatisch als dynamisch gereizte Gebärmutter war anfünglich tetanisch, also innigst an den gerade mit seiner Basis resp. mit dem Hinterhauptsloche gegen oder eigentlich auf den Muttermund aufliegenden Kopf angelagert, so dass keine Luft zwischen Schädel und Gebärmutterwand eindringen konnte, denn sonst wäre ja unbedingt früher oder später eine Physometral) entstanden, was aber eben deshalb nicht eintrat, weil die Gebärmutter sich offenbar auch weiter so permanent um den Kopf angeschmiegt rückgebildet haben musste, und auf diese Weise die durch das Hinterbauptsloch hervorsickernde faulige Gehirmmasse mit den übrigen breïg zerfallenen Weichtheilen am kürzesten Wege abzufliessen vermochte, ohne eben mit der Innenfläche der Gebärmutter in Berühıung kommen zu können.

1) Valenta: Ueber Tympanitis uteri. Wochenblatt der Zeitschrift der Gesellschaft der Aerzte zu Wien 1857, TII. Jahrgang. 
So war factisch schliesslich fast nur noch der skelettirte Schädel zurückgeblieben, denn thatsächlich wurden bei der Operation so zu sagen keine Weichtheile, sondern nur mehr die macerirten Knochen vorgefunden, von der Kopfhaut waren nur kleine Fetzen aufindbar an und mit den entfernten Knochenfragmenten.

Der Hauptgrund, dass Septicaemie trotz alledem nicht entstand, dürfte jedoch auch maassgebend in dem Verhalten der Placenta zu suchen sein, nämlich: meines Erachtens scheint durch Zug an der Nabelschnur der gegen den Gebärmutterkörper, also höher gelegene Theil des Mutterkuchens zufällig entfernt worden zu sein, und die zurückgebliebene untere Partie hat den Charakter eines fibrinösen - oder Placentarpolypen ${ }^{1}$ ) angenommen, Beweis dessen das frische Aussehen der entfernten Placentarreste, und so wurde durch die innige Anlagerung an den Kopf und die gleichzeitig begimnende puerperale Involution begïnstigt eine Aufsaugung von jauchigem Secrete hintangehalten, i. e. eine Endometritis septica vermieden, allerdings ein reiner Zufall.

Bezüglich der raschen Rückbildung der vollständig entleerten Gebärmutter möchte ich es als ein Analogon des gleichen Verhaltens bezeichnen, welches dieses Organ kundgiebt, wenn z. B. behufs Fntfernung eines Fibroids und dergleichen Dilatationen vorgenommen werden, wo dann im negativen Falle bekanntlich stets auffällig rasch der status quo ante rückkehrt, oder im positiven Falle, i. e. nach wirklich vollbrachter Entfernung eines Neugebildes, trotzdem auch keine dynamische Thätigkeit dabei mit half, sich die Gebärmutter in sehr kurzer Zeit normal involvirt zeigt.

Die kritische Ausnutzung dieses Falles in geburtshüflichoperativer Hinsicht dürfte keinen Schwierigkeiten unterliegen. Das einzig Richtige wäre gewesen: warten, den Krampf dynamisch beheben, und alsdann, den Rumpf als Hebel vernünftig benützend, wäre sicherlich die Extraction glücklich gelungen; satis sapienti:

Was die Literatur derartiger Fälle anbelangt, so habe ich, soweit mir selbe zugänglich ist, ausser dem Freund'schen Falle keinen ähnlichen vorgefunden, und wäre somit mein Fall diesbezüglich als Zwilling des Freund'schen Falles mit Recht zu

1) Valenta: Ueber puerperale Metrorrhagie infolge eines fibrösen Polypen - Memorabilien, Jahrgang 1869. 
bezeichnen, und demgemäss erscheint auch dessen detaillirte Veröffentlichung gerechtfertigt. Nicht umhin kann und muss ich jedoch bei dieser Gelegenheit ein warnend Halt! an die geburtshülflichen Antiseptiker-Ultras in Betreff der prophylactischen Desinfection Gebärender und Wöchnerinnen richten. Ich erinnere an die Vorschläge, bei jeder Geburt den Dampfspray in Anwendung zu ziehen, jeder Wöchnerin ohne Unterschied, ob gesund oder krank, die Gebärmutterhöhle zu irrigiren - - das heisst doch, aufrichtig gesagt, das Kind mit dem Bade ausgiessen in medio virtus! - Ich habe meiner Arbeit denselben Titel wie Freund gegeben, und glaube dieselbe, mutatis mutandis, trotz Carbol, Thymol, Salicyl, Jodoform u. dergl., also trotzdem, dass sich seitdem die Infectionstheorie und deren vorbeugende Behandlung gar sehr vervielfacht resp. verbessert haben, ebenso am besten mit Freund's Worten zu schliessen, nämlich: alle Infectionstheorien entsprechen vielen, aber nicht allen Thatsachen, sie sind bis dahin noch immer Hypothesen, die ihre Berechtigung haben, und wenn es auch nur darum wäre, dass sie ein nicht genug zu beherzigendes Moment in der Behandlung der Schwangeren, Gebärenden und Wöchnerinnen aufs Neue urgiren und zur unverbrüchlichen Regel gestempelt haben: die scrupulöseste Sauberkeit und die grösste Vorsicht bei den manuellen und instrumentellen Eingriffen in den Genitalapparat.

Diesen Grundsatz allgemein durchgeführt resp. eingebürgert zu haben, darin liegt in erster Linie das eigentliche unsterbliche Verdienst Lister's - ob mit Carbolsäure u. dergl. oder ohne, daruiber sind die Acten heute noch nicht geschlossen, und ob sie je geschlossen werden? - das wissen die Götter! und somit möchte ich sine ira et studio zum Schlusse nochmals ausrufen, jedoch nicht unerhört, wie die Stimme des Rufenden in der Wüste, ,halten wir Gynäkologen im engsten und weitesten Sinne des Wortes an dem Axiome fest: Schwangerschaft, Geburt und Wochenbett sind physiologische Zustände und dürfen unter keiner Bedingung als solche ein Feld der extravagantesten Experimentirung abgeben, sondern eben bis an die äussersten Grenzen ein physiologisches noli me tangere sein und bleiben"; - dixi et salvavi animam meam! 